\title{
The LTP experiment on the LISA Pathfinder mission
}

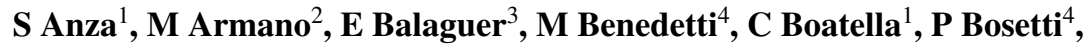 \\ D Bortoluzzi ${ }^{4}$, N Brandt ${ }^{5}$, C Braxmaier ${ }^{5}$, M Caldwell ${ }^{6}$, L Carbone ${ }^{2}$, \\ A Cavalleri $^{2}$, A Ciccolella ${ }^{3}$, I Cristofolini ${ }^{4}$, M Cruise ${ }^{7}, \mathrm{M} \mathrm{Da} \mathrm{Lio}^{4}$, \\ K Danzmann ${ }^{8}$, D Desiderio ${ }^{9}$, R Dolesi $^{2}$, N Dunbar ${ }^{10}$, W Fichter $^{5}$,

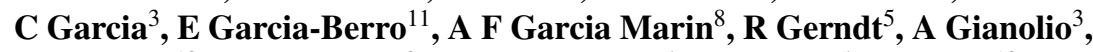 \\ D Giardini ${ }^{12}$, R Gruenagel ${ }^{3}$, A Hammesfahr ${ }^{5}$, G Heinzel $^{5}, \mathbf{J ~ H o u g h}^{13}$, \\ D Hoyland ${ }^{7}$, M Hueller ${ }^{2}$, O Jennrich ${ }^{3}$, U Johann ${ }^{5}$, S Kemble ${ }^{10}$, \\ C Killow ${ }^{13}$, D Kolbe ${ }^{5}$, M Landgraf ${ }^{14}$, A Lobo ${ }^{15}$, V Lorizzo ${ }^{9}$, D Mance ${ }^{12}$,

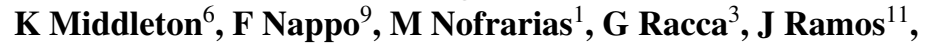

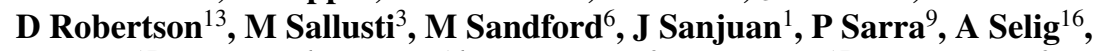

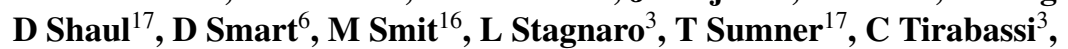

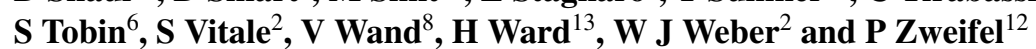

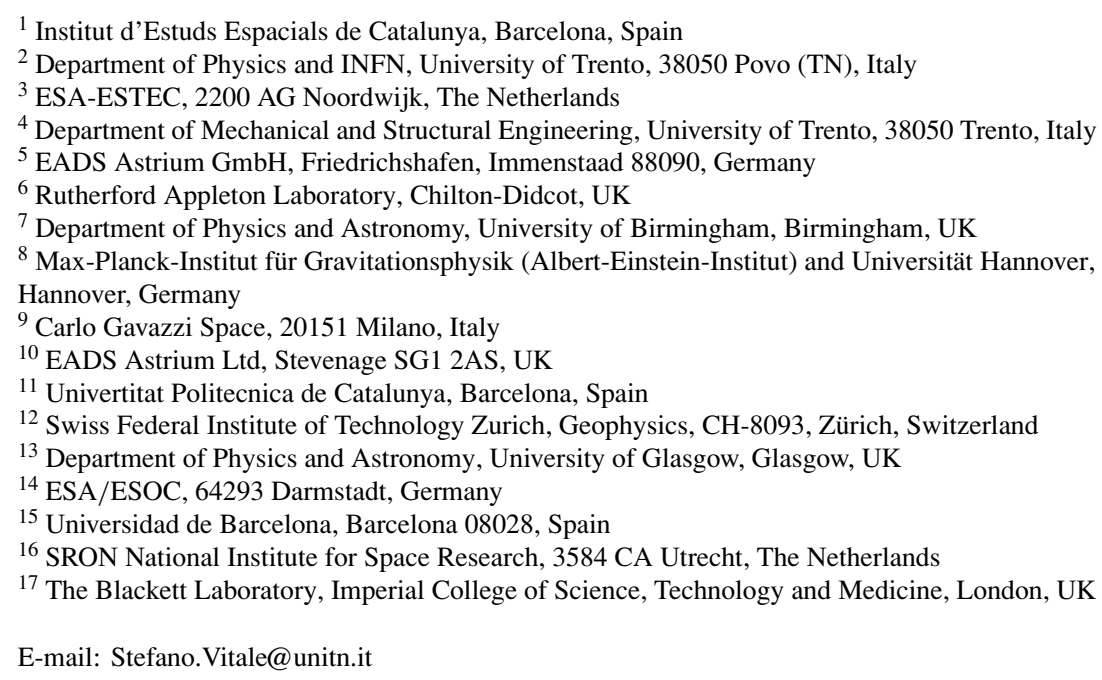

Received 31 March 2005, in final form 8 April 2005

Published 21 April 2005

Online at stacks.iop.org/CQG/22/S125

\begin{abstract}
We report on the development of the LISA Technology Package (LTP) experiment that will fly onboard the LISA Pathfinder mission of the European Space Agency in 2008. We first summarize the science rationale of the experiment aimed at showing the operational feasibility of the so-called transverse-traceless coordinate frame within the accuracy needed for LISA.
\end{abstract}


We then show briefly the basic features of the instrument and we finally discuss its projected sensitivity and the extrapolation of its results to LISA.

PACS numbers: $04.80 . \mathrm{Nn}, 95.55 . \mathrm{Ym}$

(Some figures in this article are in colour only in the electronic version)

\section{Introduction}

Our very concept of the detection of gravitational wave by an interferometric detector like LISA $[1,2]$ is based on the operative possibility of realizing a transverse and traceless (TT) coordinate frame [3].

In this kind of coordinate frame, despite the presence of the ripple in spacetime curvature due to the gravitational wave, a free particle initially at rest remains at rest, i.e. its space coordinates do not change in time, and the proper time of a clock sitting on the particle coincides with the coordinate time.

Despite the co-moving nature of such a frame, the distances among particles at rest change in time because of the change of the metric tensor, and this time variation can be detected by the laser interferometer.

Indeed a laser beam travelling back and forth between two such particles along an axis $x$ normal to the direction $z$ of the gravitational wave propagation is subject to a phase shift $\delta \theta(t)$ whose time derivative is given by [3]

$$
\frac{\mathrm{d} \delta \theta}{\mathrm{d} t}=\frac{\pi c}{\lambda}\left[h_{+}\left(t-\frac{2 L}{c}\right)-h_{+}(t)\right],
$$

where $h_{+}$is the usual definition [3] for the amplitude of the wave, $L$ is the distance between the particles and $\lambda$ is the wavelength of the laser. Furthermore, the $x$-axis has been used to define the wave polarization, so that the phase shift is only contributed by $h_{+}$and $t$ is the time at which light is collected and the frequency shift is measured.

As all this holds within a linearized theory, small effects superimpose and harmonic analysis can be applied. As a consequence, secular gravitational effects at frequencies much lower than the observation bandwidth $\left(f<10^{-4} \mathrm{~Hz}\right)$ do not matter. A TT coordinate frame may then in principle be defined just for the frequencies of relevance, letting the particles used to mark the frame change their coordinates at lower frequencies because of their motion within the gravitational field of the solar system.

If the particles are not at rest in the TT frame, i.e. if their space coordinates change in time, then obviously their distances will change also because of this motion. If the particles still move slowly relative to light, their relative motion does not affect the TT construction but competes with the signal in equation (1) by providing a phase shift:

$$
\delta \theta(t)=\frac{2 \pi}{\lambda}\left\{x_{1}(t)+x_{1}\left(t-\frac{2 L}{c}\right)-2 x_{2}\left(t-\frac{L}{c}\right)\right\}
$$

where $x_{1}$ is the coordinate of the particle sending and collecting the laser beam, while $x_{2}$ is that of the particle reflecting the light. Here coordinates are components along the laser beam and the phase shift is calculated to first order in $v / c$.

At measurement frequencies much lower than $c / L$ equation (2) gives obviously

$$
\delta \theta(t) \approx \frac{4 \pi}{\lambda} \Delta L(t) \quad \text { with } \quad \Delta L(t)=x_{1}(t)-x_{2}(t) .
$$


If all coordinates may be assumed as joint stationary random processes, the phase shift in equation (3) has a power spectral density (PSD)

$$
\begin{aligned}
S_{\delta \theta}(\omega)= & \frac{16 \pi^{2}}{\lambda^{2}}\left\{S_{\Delta L}(\omega)\left[1-2 \sin ^{2}\left(\frac{\omega L}{2 c}\right)\right]\right. \\
& \left.+8 \sin ^{2}\left(\frac{\omega L}{2 c}\right)\left[S_{x_{2}}(\omega)-\cos \left(\frac{\omega L}{c}\right) S_{x_{1}}(\omega)\right]\right\} \\
\approx & \frac{16 \pi^{2}}{\lambda^{2}} S_{\Delta L}(\omega)
\end{aligned}
$$

where $S_{\Delta L}(\omega), S_{x_{2}}(\omega)$ and $S_{x_{1}}(\omega)$ are the PSD of the related quantities at angular frequency $\omega$, and the rightmost approximate equality holds for $|\omega| L / c \ll 1$.

In a TT frame, and at low velocities, the motion of proof masses can only be caused by forces that are not due to the gravitational wave. Equation (4) then becomes

$$
S_{\delta \theta} \approx \frac{16 \pi^{2}}{\lambda^{2}} \frac{S_{\Delta F}}{\omega^{4} m^{2}}
$$

where $S_{\Delta F}$ is the spectral density of the difference of force between the proof masses.

Thus to show that a TT system can indeed be constructed with free orbiting particles, one needs to preliminarily show that non-gravitational forces on proof masses, or even locally generated gravitational forces, can be suppressed to the required accuracy in the measurement bandwidth.

The interferometer measurement noise will also compete with the gravitational signal in equation (1). This noise is usually expressed as an equivalent optical path fluctuation $\delta x$ for each passage of the light through the interferometer arm. For such an optical path fluctuation, our single-arm interferometer would suffer a phase shift

$$
\delta \theta(t) \approx \frac{2 \pi}{\lambda} \delta x(t)
$$

each way. As a consequence, if the PSD of $\delta x$ is $S_{x}$, this noise source would add an equivalent phase noise

$$
S_{\delta \theta} \approx 2 \frac{4 \pi^{2}}{\lambda^{2}} S_{x} .
$$

In LISA, the targeted sensitivity [1] requires that $S_{\Delta F}^{1 / 2}(\omega) / m^{2} \leqslant \sqrt{2} \times 3 \times 10^{-15}\left(\mathrm{~m} \mathrm{~s}^{-2}\right) \mathrm{Hz}^{-1 / 2}$ for a frequency $f$ above $f>0.1 \mathrm{mHz}$. The corresponding requirement for the interferometer is a path-length noise of $\ldots S_{x}^{1 / 2} \leqslant 20 \mathrm{pm} \mathrm{Hz}^{-1 / 2}$. With these figures, the noise in equation (5) and that in equation (7) cross at $\approx 3 \mathrm{mHz}$ thus allowing to relax the requirement for $\Delta F / m$ to

$$
\begin{aligned}
S_{\Delta F / m}^{1 / 2} & \leqslant \sqrt{2} \times 3 \times 10^{-15} \frac{\mathrm{m}}{\mathrm{s}^{2} \sqrt{\mathrm{Hz}}} \sqrt{1+\left(\frac{f}{3 \mathrm{mHz}}\right)^{4}} \\
& \approx 4.2 \times 10^{-15} \frac{\mathrm{m}}{\mathrm{s}^{2} \sqrt{\mathrm{Hz}}}\left[1+\left(\frac{f}{3 \mathrm{mHz}}\right)^{2}\right] .
\end{aligned}
$$

The requirement in equation (8) needs to be qualified. Limiting the noise in equation (4) by a requirement just for the differential force noise becomes inaccurate at frequencies above some 3-4 mHz. However, if the velocity fluctuations of the two proof masses are independent, this approach represents a worst case one. This is also the case for a partly correlated noise, provided that correlation is assumed to work in the worst direction, i.e. by mimicking differential motion. 
The focus of the above discussion has been in terms of coordinate frames. One can however restate these performance requirements in terms of coordinate-independent, or gaugeinvariant quantities, i.e. in terms of the curvature tensor $R_{\mu \nu \sigma}^{\lambda}$ only.

For a gravitational wave, the curvature tensor is equal, in the Fourier domain, to

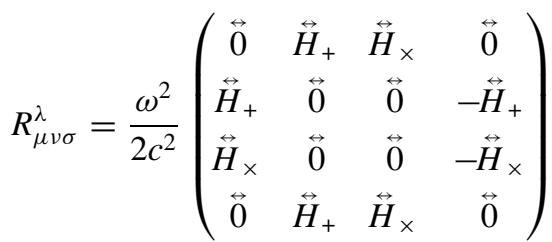

with

$$
\stackrel{\leftrightarrow}{H}_{+}=\left(\begin{array}{cccc}
0 & -h_{+}(\omega) & -h_{\times}(\omega) & 0 \\
h_{+}(\omega) & 0 & 0 & -h_{+}(\omega) \\
h_{\times}(\omega) & 0 & 0 & -h_{\times}(\omega) \\
0 & h_{+}(\omega) & h_{\times}(\omega) & 0
\end{array}\right)
$$

and

$$
\stackrel{\leftrightarrow}{H}_{\times}=\left(\begin{array}{cccc}
0 & -h_{\times}(\omega) & h_{+}(\omega) & 0 \\
h_{\times}(\omega) & 0 & 0 & -h_{\times}(\omega) \\
-h_{+}(\omega) & 0 & 0 & h_{+}(\omega) \\
0 & h_{\times}(\omega) & -h_{+}(\omega) & 0
\end{array}\right) .
$$

For $|\omega| L / 2 \ll 1$ and for an optimally oriented $(\phi=0)$ interferometer arm the phase shift in equation (1) can then be written as

$$
\delta \theta(\omega) \approx \frac{2 \pi}{\lambda} L h_{+}(\omega) \approx \frac{2 \pi}{\lambda} 2 c^{2} L \frac{R(\omega)}{\omega^{2}}
$$

with $R$ the generic component of the curvature tensor.

By comparing equation (11) with equation (8) one can recast the differential force noise as an effective curvature noise with spectrum:

$$
S_{R}^{1 / 2}(\omega) \approx \frac{1}{c^{2} L} S_{\Delta F / m}^{1 / 2} .
$$

Thus to achieve its science goals, LISA must reach a curvature resolution of order $10^{-41} \mathrm{~m}^{-2} \mathrm{~Hz}^{-1 / 2}$ or of $10^{-43} \mathrm{~m}^{-2}$ for a signal at $0.1 \mathrm{mHz}$ integrated over a cycle. This figure may be compared with the scale of the curvature tensor due to the gravitational field of the Sun at the LISA location of $\approx 10^{-30} \mathrm{~m}^{-2}$.

The aim of the LISA Pathfinder mission of the European Space Agency (ESA) is to demonstrate that indeed a TT frame may be constructed by using particles nominally free orbiting within the solar system, with accuracy relevant for LISA. Specifically within the LISA Technology Package (LTP) ${ }^{18}$, two LISA-like proof masses located inside a single spacecraft are tracked by a laser interferometer. This minimal instrument is deemed to contain the essence of the construction procedure needed for LISA and thus to demonstrate its feasibility.

This demonstration requires two steps.

- Firstly, based on a noise model $[4,5]$, the mission is designed so that any differential parasitic acceleration noise of the proof masses is kept below the requirements. For the

18 The LTP is a collaboration between ESA, and the space Agencies of Germany (DLR), Italy (ASI), The Netherlands (SRON), Spain (MEC), Switzerland (SSO) and United Kingdom (PPARC). In addition, France (CNES/CNRS) is in the process of joining the team. 
LTP these requirements are relaxed to $3 \times 10^{-14} \mathrm{~m} \mathrm{~s}^{-2} \mathrm{~Hz}^{-1 / 2}$, a factor $\approx 7$ larger than what is required in LISA. In addition, this performance is only required for frequencies larger than $1 \mathrm{mHz}$. This relaxation of performance is accepted in view of cost and time saving.

As for both LISA and the LTP, this level of performance cannot be verified on the ground due to the presence of the large Earth gravity, the verification relies mostly on the measurements of key parameters of the noise model of the instrument [6-10]. In addition, an upper limit to all parasitic forces that act at the proof-mass surface (electrostatics and electromagnetics, thermal and pressure effects, etc) has been established and keeps being updated by means of a torsion pendulum test bench [7, 11, 12]. In this instrument a hollow version of the proof mass hangs from the torsion fibre of the pendulum so that it can freely move in a horizontal plane within a housing which is representative of flight conditions. An equivalent differential acceleration noise of $\approx 3 \times 10^{-13} \mathrm{~m} \mathrm{~s}^{-2} \mathrm{~Hz}^{-1 / 2}$ [7] has been measured.

- Secondly, once in orbit the residual differential acceleration noise of the proof masses is measured. The noise model $[13,14]$ predicts that the contributions to the total PSD fall into three broad categories.

(i) Noise sources whose effect can be identified and suppressed by a proper adjustment of selected instrument parameters. An example of this is the force due to residual coupling of proof masses to the spacecraft. By regulating and eventually matching, throughout the application of electric field, the stiffness of this coupling for both proof masses, this source of noise can be first highlighted, then measured, and eventually suppressed [14].

(ii) Noise sources connected to measurable fluctuations of some physical parameter. Forces due to magnetic fields or to thermal gradients are typical examples. The transfer function between these fluctuations and the corresponding differential proof-mass acceleration fluctuations will be measured by purposely enhancing the variation of the physical parameter under investigation [14] and by measuring the corresponding acceleration response. For instance, the LTP carries magnetic coils to apply comparatively large magnetic field signals and heaters to induce timevarying thermal gradients. In addition, the LTP also carries sensors to measure the fluctuation of the above physical disturbances while measuring the residual differential acceleration noise in the absence of any applied perturbation. Examples of these sensors are magnetometers and thermometers to continue with the examples above. By multiplying the measured transfer function by the measured disturbance fluctuations, one can generate an expected acceleration noise data stream to be subtracted from the main differential acceleration data stream. This way the contributions of these noise sources are suppressed and the residual acceleration PSD decreased. This reduction is obtained without requiring expensive magnetic 'cleanliness' or thermal stabilization programmes.

(iii) Noise sources that cannot be removed by any of the above methods. The residual differential acceleration noise due to these sources must be accounted for [14]. To be able to do the required comparison, some of the noise model parameters must and will be measured in-flight. One example for all, the charged particle flux due to cosmic rays will be continuously monitored by a particle detector.

The result of the above procedure is the validation of the noise model for LISA and the demonstration that no unforeseen source of disturbance is present that exceeds the residual 


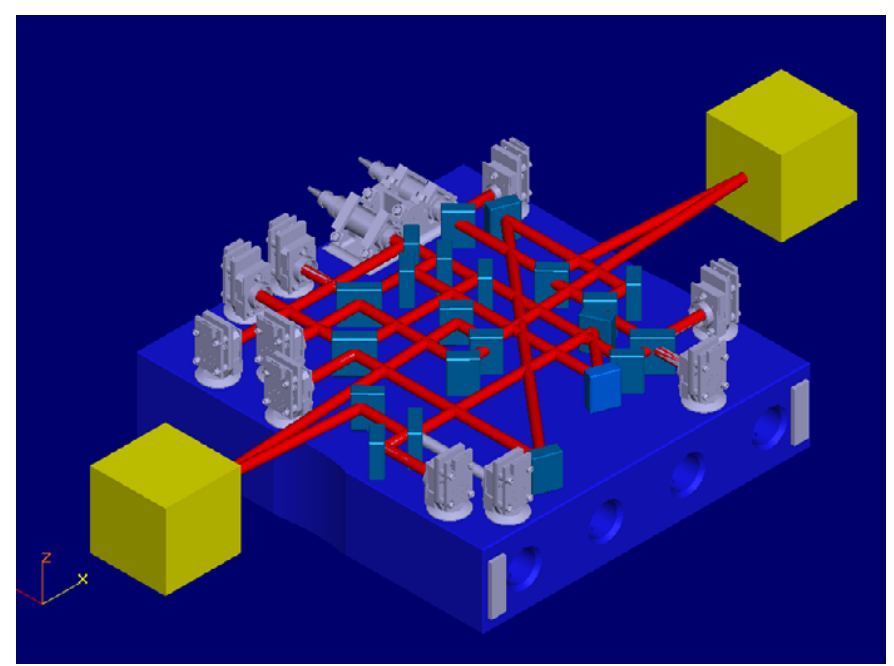

Figure 1. The concept of the LTP: the distance between two cubic, free-floating proof masses is measured by a heterodyne laser interferometer. The proof-mass centres' nominal separation is $L=376 \mathrm{~mm}$, the proof-mass size is $46 \mathrm{~mm}$ and the mass value is $1.96 \mathrm{~kg}$.

uncertainty on the measured PSD. The following sections, after describing some details of the experiment, will discuss the expected amount of this residual uncertainty.

\section{The LISA Technology Package experiment}

The basic scheme of the LTP has been described in [2] and is shown in figure 1: two freefloating proof masses are hosted within a single spacecraft and the relative motion along a common sensitive axis, the $x$-axis, is measured by means of a laser interferometer. The proof masses are made of a gold-platinum, low magnetic susceptibility alloy, have a mass of $m=$ $1.96 \mathrm{~kg}$ and are separated by a nominal distance of $376 \mathrm{~mm}$. A picture of the instrument in its current stage of design and a few pictures of a prototype under development are given in figures 2-4. In the following section, we summarize the basic elements.

\subsection{The LTP instrument}

In the LTP, as in LISA, each proof mass is surrounded by a set of electrodes that are used to read out the mass position and orientation relative to the spacecraft $[11,12,15,16]$ (figure 3). This measurement is obtained as the motion of the proof mass varies the capacitances between the electrodes and the proof mass itself. The same set of electrodes is also used to apply electrostatic forces to the proof masses.

Differential capacitance variations are parametrically read out by a front-end electronics composed of high accuracy differential inductive bridges excited at about $100 \mathrm{kHz}$, and synchronously detected via a phase sensitive detector [15, 17]. Sensitivities depend on the degree of freedom. For the $x$-axis it is better than $1.8 \mathrm{~nm} \mathrm{~Hz}^{-1 / 2}$ at $1 \mathrm{mHz}$. Angular sensitivities are better than $200 \mathrm{nrad} \mathrm{Hz}^{-1 / 2}$.

Forces and torques on the proof masses required during science operation are applied through the same front-end electronics by modulating the amplitude of an ac carrier applied to the electrodes. The frequency of the carrier is high enough to prevent the application of 


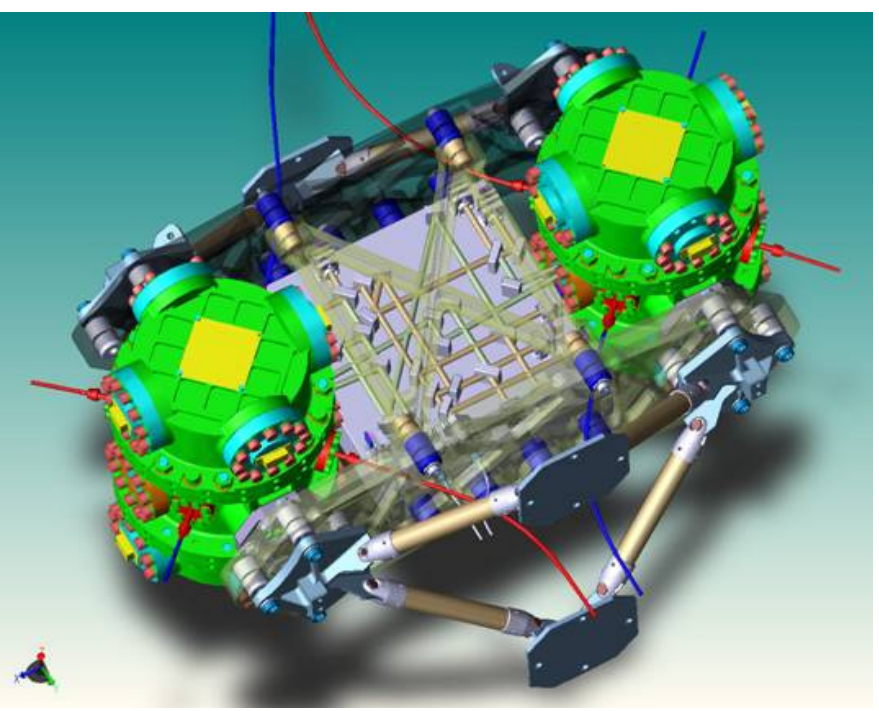

Figure 2. The LISA Technology Package. The metal chambers are the vacuum enclosures that provide autonomous ultra-high vacuum around the proof masses (non-visible). An optical bench inbetween the proof masses supports the interferometry that reads out the distance between the masses. The interferometer laser beam hits each proof mass by crossing the vacuum enclosures through an optical window. The entire supporting structure is made out of glass-ceramics for high thermo-mechanical stability. Also visible are the optical fibres that carry the UV light used for contact-less discharging of proof masses and the side struts that connect the LTP to the spacecraft.

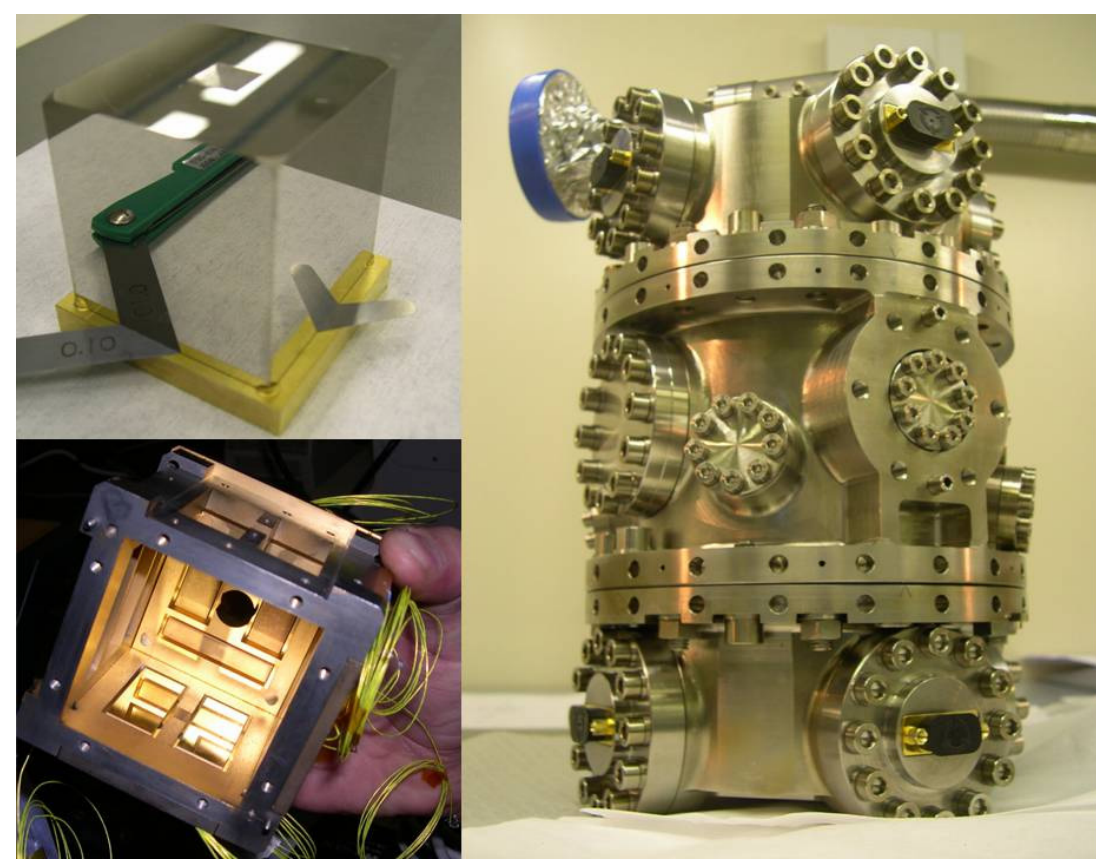

Figure 3. A prototype of the gravity reference sensor under development. Top left: the goldplatinum proof mass. Bottom left: the electrode housing carrying the gold-coated ceramics electrodes. Right: the vacuum enclosure. The optical windows for the laser light are substituted by plane flanges. 


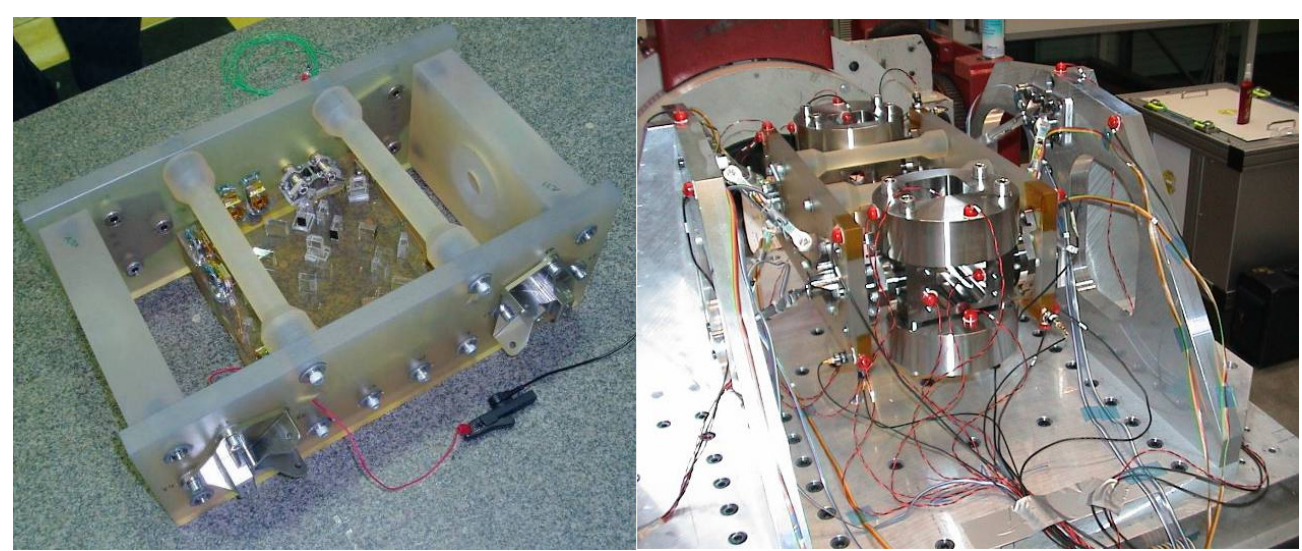

Figure 4. Left: a prototype of the optical bench under development. Right: vibration test of the optical bench with two dummies of the gravity reference sensors to simulate the launch loads.

unwanted forces by mixing with low-frequency fluctuating random voltages. The front-end electronics is also used to apply all voltages required by specific experiments.

Each proof mass, with its own electrode housing, is enclosed in a high vacuum chamber which is pumped down to $10^{-5} \mathrm{~Pa}$ by a set of getter pumps. The laser interferometer light passes through the vacuum chamber wall through an optical window.

As the proof mass has no mechanical contact to its surrounding, its electrical charge continues to build up due to cosmic rays. To discharge the proof mass, an ultra-violet light is shone on it and/or on the surrounding electrodes [18]. Depending on the illumination scheme, the generated photoelectrons can be deposited on or taken out of the proof mass to achieve electrical neutrality.

The absence of a mechanical contact also requires that a blocking mechanism keeps the mass fixed during launch and is able to release it overcoming the residual adhesion. This release must leave the proof mass with small enough linear momentum to allow the control system described in the following to bring it at rest in the nominal operational working point.

The system formed by one proof mass, its electrode housing, the vacuum enclosure and the other subsystems is called in the following the gravity reference sensor (GRS).

The interferometer system provides the following measurements: (1) heterodyne measurement of the relative position of the proof masses along the sensitive axis. (2) Heterodyne measurement of the position of one of the proof masses (proof mass 1) relative to the optical bench. (3) Differential wave-front sensing of the relative orientations of the proof masses around the $y$-axis and the $z$-axis. (4) Differential wave-front sensing of the orientation of proof mass 1 around the $y$-axis and the $z$-axis. Sensitivities at $\mathrm{mHz}$ frequency are in the range of $10 \mathrm{pm} \mathrm{Hz}^{-1 / 2}$ for displacement and of $10 \mathrm{nrad} \mathrm{Hz}^{-1 / 2}$ in rotation.

As a light source for the heterodyne interferometry, a diode-pumped, monolithic Nd:YAG non-planar ring oscillator is used. To obtain the necessary frequency shift, the beam coming from the laser is split and each partial beam is sent through an acousto-optical modulator (AOM). The light is then delivered to the optical bench by a pair of optical fibres and fibre injectors. Quadrant photo-diodes are used for the detection of the interferometric signal, allowing us to measure yaw and pitch of the proof masses with respect to the sensitive axis. The optical components needed for the interferometer (i.e. mirrors and beam-splitters) are attached to the optical bench through hydroxyl-silicate bonding to ensure mechanical stability. 


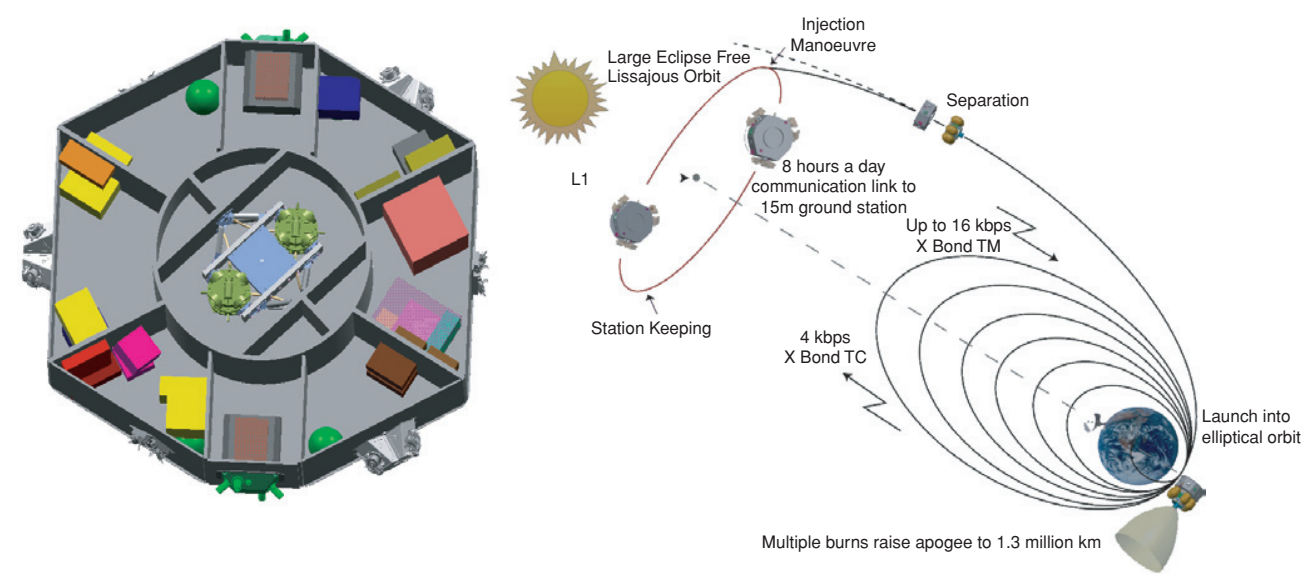

Figure 5. Left: the LTP accommodated within the central section of the LISA Pathfinder spacecraft. Right: the injection of LISA Pathfinder in the final orbit around L1.

Data acquisition, conditioning and phase measurement is performed by the interferometer front-end electronics, based largely on field programmable gate arrays (FPGA). The final processing and retrieval of the position signals from the phase measurements is performed by the LTP payload computer.

A full description of the interferometer can be found in [19].

The LTP computer also drives and reads out the set of subsidiary sensor and actuators [20] needed to apply the already mentioned selected perturbations to the proof masses and to measure the fluctuations of the disturbing fields. Actuators include coils used to generate magnetic field and magnetic field gradients and heaters to vary temperature and temperature differences at selected points of the gravity reference sensor and of the optical bench. Sensors include magnetometers, thermometers, particle detectors and monitors for the voltage stability of the electrical supplies.

The LTP will fly on LISA Pathfinder [21] in 2008. It will be hosted in the central section of the spacecraft (figure 5, left), where gravitational disturbances are minimized, and will operate in a Lissajous orbit [22] around the Lagrange point 1 of the Sun-Earth system, an environment very similar to that where the LISA spacecrafts will operate (figure 5, right).

Further details can be found in [21].

\subsection{Experiment performance: similarities and differences with LISA}

Within the LTP, as in LISA, a key element for suppressing the force disturbance is that the proof masses have no mechanical contact to the spacecraft. In addition, as forces may depend on the position of the proof masses within the spacecraft, this is kept as fixed as possible.

To fulfil both of these apparently conflicting requirements [1], the spacecraft actively follows the proof mass located within it, in a closed loop control scheme usually called the drag-free control. The position of the proof mass relative to some nominal origin is measured by means of the gravitational reference sensor. A high gain control loop tries to null this error signal by forcing the spacecraft to follow the proof mass. In order to produce the necessary force on the spacecraft, the control loop drives a set of micro-thrusters. 
With this control scheme, if the loop gain is high enough, the difference of force between two masses sitting in two different spacecraft can be calculated [2] to be

$$
\frac{\Delta F}{m}=\frac{\Delta f}{m}-\omega_{\mathrm{p} 2}^{2} \delta x_{2}+\omega_{\mathrm{p} 1}^{2} \delta x_{1}
$$

where $\Delta f$ is the difference of position-independent, fluctuating forces directly acting on proof masses, $\omega_{\mathrm{p} 1(2)}^{2}$ is the stiffness per unit mass of parasitic spring coupling of proof mass 1(2) to the spacecraft and $\delta x_{1(2)}$ is the residual jitter of the same proof mass relative to the spacecraft.

The LTP experiment uses a similar drag-free control scheme. However here both masses sit in one spacecraft and cannot be simultaneously followed by it. One of them must be forced to follow either the other one or the spacecraft, possibly within a closed loop control scheme. The small required force is applied via the capacitive actuation described above and this control loop is usually called the electrostatic suspension.

Various modes of control are envisaged [23, 24]. In the basic one the spacecraft follows one of the proof masses, say proof mass 1 , along the direction of the interferometer arm. In addition, the main interferometer read-out, i.e. the distance between the proof masses, is used as the error signal to actuate the second proof mass along the same axis. This way the distance between the proof masses is kept constant.

The laser interferometer output, at angular frequency $\omega$, is given, in the Fourier space, by

$$
\delta x_{\text {ifo }}=x_{\text {n,ifo }}+\frac{\Delta F}{\omega^{2} m}
$$

where $x_{\mathrm{n}, \text { ifo }}$ is the interferometer displacement noise and the difference of force on the righthand side includes all possible contributions.

$\Delta F / m$ is affected by the control loops. In the approximation of high drag-free gain and small parasitic stiffness, $\Delta F / m$ is given by [14]

$$
\frac{\Delta F}{m} \approx \frac{\omega^{2}}{\omega^{2}-\omega_{\mathrm{es}}^{2}(\omega)}\left\{\frac{\Delta f}{m}+\left(\omega_{\mathrm{p} 1}^{2}-\omega_{\mathrm{p} 2}^{2}\right) \delta x_{1}+\omega_{\mathrm{es}}^{2}(\omega) x_{\mathrm{n}, \text { ifo }}\right\}
$$

where $\omega_{\mathrm{es}}^{2}(\omega)$ is the gain (per unit mass) of the electrostatic suspension loop. $\Delta f$ in the parentheses is again the position-independent difference of forces that would act on the proof masses in the absence of any control loop.

The effective measurement of $\Delta F / m$ is performed by measuring the signal $\delta x_{\text {ifo }}$ or, even better, its second time derivative. By using equation (14) one gets

$$
\omega^{2} \delta x_{\mathrm{ifo}} \approx \frac{\omega^{2}}{\omega^{2}-\omega_{\mathrm{es}}^{2}(\omega)}\left\{\frac{\Delta f}{m}+\left(\omega_{\mathrm{p} 1}^{2}-\omega_{\mathrm{p} 2}^{2}\right) \delta x_{1}+\omega^{2} x_{\mathrm{n}, \mathrm{ifo}}\right\} .
$$

Equation (16) brings a remarkable similarity to equation (13), thus suggesting that indeed the basic concept of the experiment is sound. However, there are a few remarks that need to be made.

In the LTP both proof masses are spring-coupled to the same spacecraft and both feel then the relative jitter between this one and the drag-free reference proof mass 1 . Some care must then be used to avoid that, by getting $\omega_{\mathrm{p} 1}^{2} \simeq \omega_{\mathrm{p} 2}^{2}$ a substantial residual jitter $\delta x_{1}$ becomes unobservable, thus yielding an estimate of the noise in equation (13) that is too small. This is easily avoided by a detailed sequence of measurements for both $\omega_{\mathrm{p} 1}^{2}$ and $\omega_{\mathrm{p} 2}^{2}$ that has been described in [14].

Correlation of disturbances on different proof masses may play a different role in LISA and in the LTP. In LISA proof masses within the same interferometer arm belong to different spacecraft and are located $5 \times 10^{9} \mathrm{~m}$ apart. The only correlated disturbances one can think of are connected to the coupling to the Sun: magnetic field fluctuations, fluctuation of the 
flux of charged particles in solar flares and the fluctuation of solar radiation intensity that may induce correlated thermal fluctuations in distant spacecraft. These correlations will only slightly affect the error budget and will have no profound consequences on the experiment itself.

In the LTP all sources of noise that share the same source for both proof masses are correlated. Magnetic noise generated onboard the spacecraft, thermal fluctuations and gravity noise due to thermo-elastic distortion of spacecraft constitute a few examples. The major concern with correlated noise is that, by affecting the proof masses the same way, it might subtract from the differential measurement. This subtraction would not happen in LISA and thus would cause an optimistic underestimate of the total noise. This possibility can reasonably be avoided for almost all candidate effects: magnetic disturbances are quadratic functions of the field and can then be modulated by purposely applying asymmetric fields and gradient to different proof masses (that will anyhow have susceptibility and remnant moments only matched not better than $50 \%$ ). Sensitivity to thermal gradient can be modulated by independently adjusting the static temperature of each proof mass. Similar strategies of intentional mismatch of the response function of the two proof masses can be devised for almost all disturbances.

An exception is constituted by the gravitational noise for which the response, due to the equivalence principle, cannot be changed. However, realistic assumptions about thermal distortion make the event of a gravity fluctuation affecting both proof masses with the same force along the $x$-axis very unlikely.

In addition, LISA Pathfinder also carries the independent NASA-provided ST-7 technology package [25], based on the same concept as the LTP, which will operate for part of the mission jointly with the LTP. The possibility that these gravitational disturbances cancel exactly and simultaneously on both instruments is even more unlikely, if not impossible. Note that the joint operation between LTP and ST-7 will also allow detection some of the correlated disturbances discussed above.

The presence of the electrostatic suspension multiplies the forces in equation (16) by the transfer function $\omega^{2}\left[\omega^{2}-\omega_{\mathrm{es}}^{2}(\omega)\right]^{-1}$. Though the numerical algorithm behind $\omega_{\mathrm{es}}^{2}(\omega)$ is entirely known, this gain also includes the conversion from a force command issued by a computer to actual voltages and forces on the proof masses, thus posing a calibration issue if $\left|\omega_{\mathrm{es}}^{2}(\omega)\right| \gtrsim \omega^{2}$. If instead $\left|\omega_{\mathrm{es}}^{2}(\omega)\right| \ll \omega^{2}$, then the $\omega^{2} \delta x_{\text {ifo }}$ is a faithful representation of the force signal. This last limit is then accurate and can be used for calibration, but it brings about the disadvantage that $\omega_{\mathrm{es}}^{2}(\omega)$ must be kept small at all relevant frequencies, thus producing long and slightly impractical relaxation time constants in the electrostatic suspension loop. As an alternative, within the joint operation with ST-7, a calibration procedure is envisaged where a comparatively large motion of one of the proof masses of the ST-7 package is used to generate an oscillating gravitational field on the LTP proof masses. This will allow an independent absolute calibration of forces onboard at better than $1 \%$.

Finally, equation (16) shows that the read-out noise contributes the feedback force $\omega^{2} x_{\mathrm{n} \text {,ifo }}$ that is not present in the case of LISA. This is discussed in the following section.

\subsection{Experiment performance: sensitivity}

As an instrument to measure $\Delta F / m$, the LTP is then limited by the laser interferometer noise. When $\left|\omega_{\text {es }}^{2}(\omega)\right| \ll \omega^{2}$ the signal is

$$
\omega^{2} \delta x_{\mathrm{ifo}} \approx \frac{\Delta f}{m}+\left(\omega_{\mathrm{p} 1}^{2}-\omega_{\mathrm{p} 2}^{2}\right) \delta x_{1}+\omega^{2} x_{\mathrm{n}, \mathrm{ifo}} .
$$


Equation (17) shows that, as in LISA, the laser interferometer noise converts into an effective force noise according to

$$
S_{\Delta F / m}^{1 / 2}(\omega) \approx \omega^{2} S_{\mathrm{n}, \mathrm{ifo}}^{1 / 2}(\omega)
$$

For the LTP, the laser interferometer is requested to achieve a sensitivity of

$$
S_{\mathrm{n}, \text { laser }}^{1 / 2}(\omega)=\left(9 \mathrm{pm} \mathrm{Hz}^{-1 / 2}\right) \sqrt{1+\left(\frac{\omega}{2 \pi \times 3 \mathrm{mHz}}\right)^{-4}} .
$$

This noise corresponds to an equivalent force noise of

$$
S_{\Delta F / m}^{1 / 2}(f) \approx\left(3.2 \times 10^{-15} \mathrm{~m} \mathrm{~s}^{-2} \mathrm{~Hz}^{-1 / 2}\right)\left[1+\left(\frac{f}{3 \mathrm{mHz}}\right)^{2}\right]
$$

At lower frequencies an additional force noise adds up to mask the parasitic forces due to other sources. This force noise is due to the fluctuations of the gain of the electrostatic suspension loop. Indeed the electrostatic suspension must also cope with any static force acting on the proof masses. If the force stays constant but the gain fluctuates, the feedback force fluctuates consequently, adding a noise source that is expected to limit the sensitivity at the lowest frequencies. This effect only appears in the LTP as in LISA static forces are compensated just by the drag-free loop, and no electrostatic suspension is envisaged. The largest expected source of gain fluctuations is the fluctuation of the dc voltage which is used to stabilize the actuation electronics.

A relative fluctuation of the voltage $\delta V / V$ would then produce a fluctuation of force:

$$
\frac{\delta f}{m} \approx\left(\frac{\Delta f}{m}\right)_{\text {static }} \times 2 \frac{\delta V}{V}
$$

where the factor 2 comes from the quadratic conversion from voltage to force.

For the LTP it is required that $|\Delta f / m|_{\text {static }} \leqslant 1.3 \times 10^{-9} \mathrm{~m} \mathrm{~s}^{-2}$. However to keep some margin, balancing of the gravitational field, the largest source of dc forces, is requested at one-half of the above figure and current models predict that the remaining sources of dc forces are negligible. In addition, the dc-voltage reference is required to be stable to within $S_{\delta V / V}^{1 / 2} \leqslant 2 \times 10^{-6} \mathrm{~Hz}^{-1 / 2}$ at $1 \mathrm{mHz}$. As a goal this stability should not degrade worse than $1 / f$ at lower frequencies down to $0.1 \mathrm{mHz}$. If one can achieve these goals, gain fluctuations may convert to an equivalent force PSD of

$$
S_{f_{\mathrm{fb} / \mathrm{m}}}^{1 / 2}(f) \approx 1.8 \times 10^{-15} \frac{\mathrm{m} \mathrm{s}^{-2}}{\sqrt{\mathrm{Hz}}} \sqrt{1+\left(\frac{1 \mathrm{mHz}}{f}\right)^{2}} .
$$

Adding up the results in equations (22) and (20) one gets the sensitivity prediction in figure 6 .

In figure 6 we also report for comparison, besides the LISA requirements from equation (8) and the LTP maximum noise requirements, the required sensitivity for mission success for LISA, the so-called minimum mission requirements. This mission would still detect gravitational waves from merger of black holes with $3 \times 10^{5} \mathrm{M}_{\odot}$ at $z=1$ with high $\mathrm{S} / \mathrm{N}$ ratio. Furthermore, it would be able to detect and study the waves from thousands of galactic compact binaries. Finally, it would detect at SNR $>10$ one or more of the well identified galactic binaries that are usually called 'verification binaries' as everything is known about the expected gravitational wave signal. 


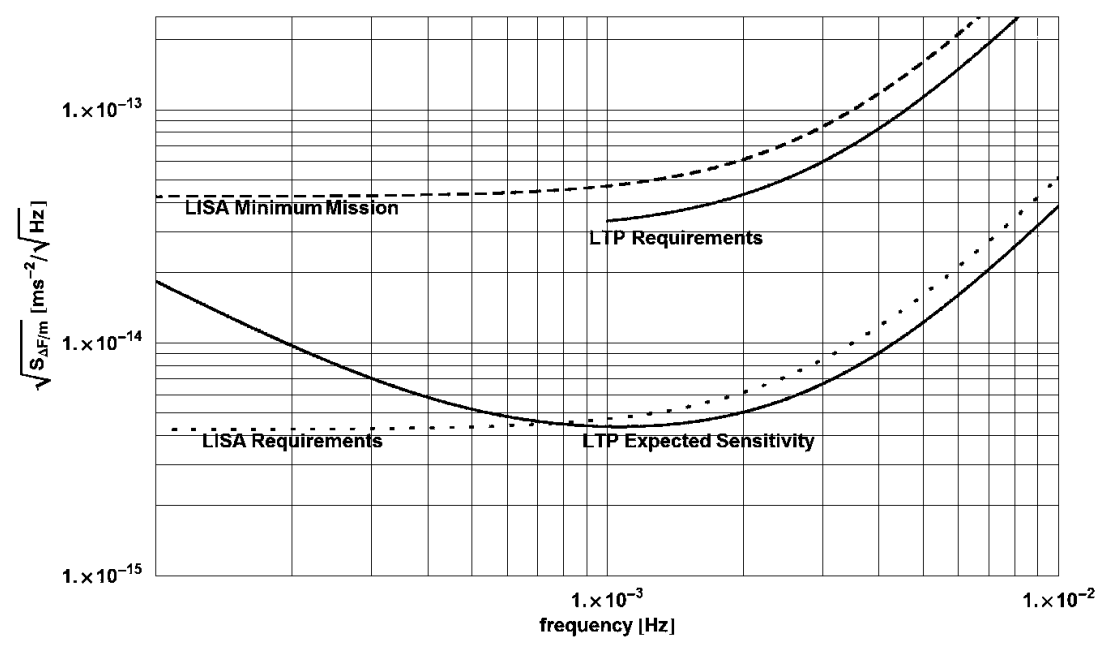

Figure 6. Lower solid curve: projected sensitivity for differential force measurement of the LTP experiment. Upper solid curve: required maximum differential acceleration noise for the LTP. Lower dotted curve: LISA requirements from equation (8). Upper, dashed curve: LISA minimum mission requirements.

\section{Concluding remarks}

Figure 6 shows that the ultimate uncertainty on the differential acceleration PSD can be potentially constrained by the LISA Pathfinder mission below a factor 5 above LISA requirements at $0.1 \mathrm{mHz}$ and near LISA requirements at $1 \mathrm{mHz}$ or above.

In addition, within the entire frequency range, the LISA Pathfinder mission will constrain the acceleration noise somewhere in the range between 1 and $10 \mathrm{fm} \mathrm{s}^{-2} \mathrm{~Hz}^{-1 / 2}$ well below the requirements of LISA minimum mission, thus strongly reducing the risk of a LISA failure.

Note that the resulting TT frame, a frame where free particles at rest remain at rest, is a very close approximation to the classical concept of inertial frame, and would indeed be inertial, within the measurement bandwidth, would it not be for the presence of the gravitational wave. Thus, LISA Pathfinder will demonstrate the possibility of building an inertial frame in a standard spacecraft orbiting the Sun on a scale of a metre in space and of a few hours in time at the above-mentioned level of absence of spurious accelerations. To our knowledge this will be at least two orders of magnitude better than what will be achieved by the GOCE mission [26] which will in turn be much better than any existing current limit.

\section{References}

[1] Bender P et al 1986 LISA Pre-Phase-A Report MPQ 208 (Munich) unpublished

[2] Vitale S et al 2002 Nucl. Phys. B (Proc. Suppl.) 110209

[3] Misner C W, Thorne K S and Wheeler J A 1973 Gravitation (San Francisco: Freeman)

[4] Stebbins R T et al 2004 Class. Quantum Grav. 21 S653-60

[5] Vitale S 2002 LISA Technology Package Architect Final Report ESTEC contract no 15580/01/NL/HB

[6] Hueller M et al 2002 Class. Quantum Grav. 19 1757-65

[7] Carbone L et al 2003 Phys. Rev. Lett. 91151101

[8] Carbone L et al 2004 Class. Quantum Grav. 21 S611-20

[9] Carbone L et al 2005 Class. Quantum Grav. 22 S509 (Preprint gr-qc/0412103)

[10] Hueller M et al 2005 Class. Quantum Grav. 22 S521 (Preprint gr-qc/0412093)

[11] Vitale S and Dolesi R 2000 AIP Conf. Proc. 523231 
[12] Cavalleri A et al 2001 Class. Quantum Grav. 18 4133-44

[13] Bortoluzzi D et al 2003 Class. Quantum Grav. 20 S89-97

[14] Bortoluzzi D et al 2004 Class. Quantum Grav. 21 S573

[15] Weber W J et al 2002 Proc. SPIE 485631

[16] Dolesi R et al 2003 Class. Quantum Grav. 20 S99-108

[17] Mance D and Zweifel P 2004 LISA Pathfinder Mission LTP_Inertial Sensor Front-End Electronics S2-ETHRS-3001 (ETH-Zurich 2004)

[18] Sumner T et al 2004 Class. Quantum Grav. 21 S597-602

[19] Heinzel G et al 2004 Class. Quantum Grav. 21 S581-7

[20] Lobo A et al 2004 Data Diagnostics System Science Requirements Document S2-IEEC-RS-3002 (IEEC, Barcelona 2004)

[21] Dunbar N, Tomkins K, Gould K, Lecuyot A, Holt T and Fichter W 2004 LISA Pathfinder System Design Synthesis Report S2.ASU.RP.2003 (EADS-ASTRIUM 2004)

[22] Landgraf M, Heckler M and Kemble S 2005 Class. Quantum Grav. 22 S487

[23] Bortoluzzi D et al 2003 Class. Quantum Grav. 20 S227-38

[24] Fichter W et al 2005 Class. Quantum Grav. 22 S139

[25] http://nmp.jpl.nasa.gov/st7/

[26] http://www.esa.int/export/esaLP/goce.html 